\title{
Gradhiva
}

GRADHIV

Revue d'anthropologie et d'histoire des arts

$33 \mid 2022$

Wampum : les perles de la diplomatie

\section{Le wampum, une tradition vivante}

Wampum. A Living Tradition

Peter Jemison, Jamie Jacobs et Michael Galban

Traducteur : Camille Joseph

\section{(2) OpenEdition}

Journals

Édition électronique

URL : https://journals.openedition.org/gradhiva/6114

DOI : 10.4000/gradhiva.6114

ISSN : 1760-849X

Cet article est une traduction de :

Wampum: A Living Tradition - URL : https://journals.openedition.org/gradhiva/6265 [en]

Éditeur

Musée du quai Branly Jacques Chirac

Édition imprimée

Date de publication : 2 février 2022

Pagination : 118-131

ISBN : 978-2-35744-133-0

ISSN : 0764-8928

\section{Référence électronique}

Peter Jemison, Jamie Jacobs et Michael Galban, « Le wampum, une tradition vivante », Gradhiva [En ligne], 33 | 2022, mis en ligne le 02 février 2022, consulté le 15 septembre 2022. URL : http:// journals.openedition.org/gradhiva/6114; DOI : https://doi.org/10.4000/gradhiva.6114 


\section{Le wampum, \\ une tradition \\ vivante}


Le présent texte est issu d'une collaboration entre les Hodinöhsö:ni' (ou Haudenosaunee), longtemps appelés Iroquois, et le musée du quai Branly-Jacques Chirac. L'histoire des relations entre les musées et les peuples autochtones est jalonnée d'erreurs d'interprétation et d'idées fausses. Connaître le savoir traditionnel des peuples autochtones est crucial pour comprendre la manière dont ils sont susceptibles d'appréhender une exposition ou un objet matériel. Dans certaines nations, par exemple celle des Hodinöhsö:ni', les porteurs culturels peuvent compter sur une tradition forte, codifiée et inscrite dans la continuité, fixant la manière d'en transmettre le contenu à la génération suivante. Grâce à cela, le cœur de la culture des Hodinöhsö:ni' est resté intact pendant plusieurs siècles. Chez les Hodinöhsö:ni', certains individus ont réussi à développer, grâce aux rôles qu'ils ont joués au sein de la communauté et à leur détermination personnelle, une compréhension approfondie de la vision du monde, de la culture et de l'histoire de leur peuple. La réalisation de travaux historiques et l'émergence d'une communauté de chercheurs ont en outre permis d'alimenter l'histoire orale de ces traditions. C'est à partir du moment où la recherche académique et les voix traditionnelles se mêlent qu'on peut commencer à entrevoir un avenir où toutes ces connaissances accumulées profitent à tous.

1. Nom d'un puissant chef onondaga des temps mythiques (NdE).

2. Le mythe de la fondation de la Ligue iroquoise met en scène le héros culturel Hiathawa qui, avec le soutien du chef Dekanawidah (Deganawi:dah), le Grand Pacificateur porteur d'un message de paix pour unir les Cinq Nations, parvient à unifier les cing nations iroquoises. Pour parvenir a iroquoises. Pour parvenir à cette unité, les quatre nation Mohawk, Oneida, Cayuga et Seneca font force commune pour convaincre le chef despote Onondaga Atotarho (Thadoda:ho?) d'intégrer la Confédération. Celui-ci refuse plusieurs fois avant d'accepter de faire de sa nation le centre de la Ligue et d'avoir à sa charge la responsabilité du feu du conseil communal ou grand feu. Dekanawidah

(Deganawi:dah) pacifie

Atotarho (Thadoda:ho?) par l'offrande de wampum blanc qui est dès lors étroitement associéà toutes les manifestations politiques de la Ligue iroquoise (Hale 1881: 10 sqq.).

\section{LE WAMPUM - PETER JEMISON}

Il est sans doute difficile d'imaginer qu'il existait autrefois un système binaire utilisant les coquilles de la palourde (Mercenaria mercenaria) et du bulot (Sinistrofulgur perversum), mais ces coquillages forment bien un langage pour les Hodinöhsö:ni', ou Confédération des Iroquois. La partie violette de la palourde, qu'on dit parfois noire, et la partie blanche du bulot étaient transformées en perles par les nations côtières de langue algonquienne, puis assemblées en colliers de wampum par les Hodinöhsö:ni' de lanǵue iroquoienne, établis à l'intérieur des terres.

Ces colliers servaient d'aide-mémoire permettant de se souvenir de messages particulièrement importants. Ce sont les traces visuelles d'une idée qui peut ainsi être «lue». Les hommes hodinöhsö:ni' apprenaient à mémoriser le sens des colliers et à les lire lorsque des accords ou des messages étaient échangés avec les peuples ne parlant pas le hodinöhsö:ni'. Les wampums étaient ainsi utilisés lors de la signature des traités. C'est notamment le cas du collier wampum appelé la «George Washington Covenant Belt», plus connue sous le nom de George Washington Belt. Ce collier est lié au traité de paix et d'amitié de Canandaiǵua négocié en 1794 dans le sillage de la Révolution américaine, entre Timothy Pickering, représentant des États-Unis, et les chefs des Six Nations hodinöhsö:ni'. Le collier fut commandité par George Washington et réalisé par deux femmes oneida vers 1792.
Une reproduction de ce collier est aujourd'hui portée le 11 novembre de chaque année par un chef hodinöhsö:ni'à Canandaigua à l'occasion de la commémoration du traité de Canandaigua ou traité Pickering.

Le collier de wampum Ha:yëwënta' ou Hiawatha Belt ${ }^{1}$ est un autre exemple de collier célébrant un événement important, ici la Grande Loi qui réunit les Cinq Nations iroquoises - Mohawk, Oneida, Onondaga, Cayuga et Seneca - en une confédération. Nos nations sont unies par un même message de paix et d'amitié, symbolisé par la ligne blanche qui les relie. Au centre du wampum, sous la forme d'un arbre, se trouve la nation Onondaǵa, à droite les Oneida et Mohawk, et à gauche les Cayuǵa et Seneca. Les nations sont représentées sur un fond de perles violettes symbolisant l'époque du conflit et le moment où le message de paix fut apporté par notre Peacemaker [Pacificateur] ${ }^{2}$ pour unifier les Cinq Nations. La sagesse ancestrale de notre peuple doit continuer à vivre. Nous avons créé des formes modernes de wampum afin que cette tradition puisse perdurer. Ce collier de wampum a par exemple été reproduit sous forme de drapeau pour les Hodinöhsö:ni'. Celui-ci est désormais un symbole de la Confédération des Iroquois qu'on retrouve sur des autocollants, des casquettes, des T-shirts ou encore sur les tenues des équipes iroquoises de lacrosse. Mais cela ne suffisant pas, nos chefs ont décidé qu'il fallait de nouveau faire entendre le message de paix afin de guider les futures générations. La Grande Loi a ainsi été lue à travers tous les territoires qui composent nos nations. 


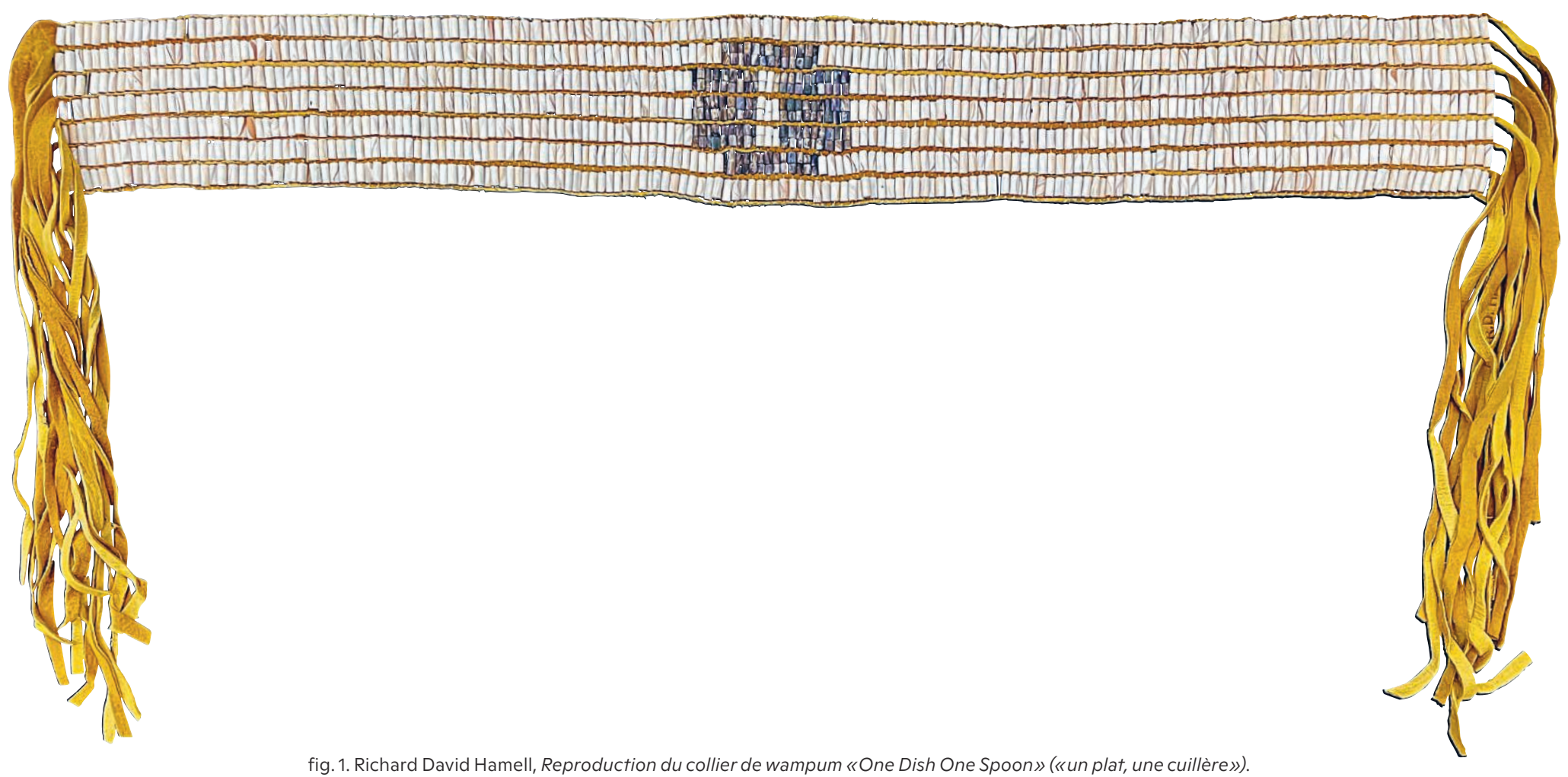

Perles en plastique imitant le wampum, cuir, fibres végétales, photographie, $8,3 \times 72,4 \mathrm{~cm}$. Avec l'aimable autorisation de Peter Jemison.

L'image de la Ha:yëwënta' Belt, devenue le symbole de la souveraineté hodinöhsö:ni' et créée avant que les Tuscarora ne rejoignent la Confédération en 1723 , unit aujourd'hui six nations par un même message de paix.

Un collier de wampum a également été créée pour l'équipe irlandaise de lacrosse. En effet, l'équipe nationale des Iroquois n'a pas été invitée à participer aux Championnats du monde de 2022. En soutien, l'équipe d'Irlande a alors décidé de céder sa place aux joueurs iroquois pour permettre aux Hod inöhsö:ni' d'être représentés durant les jeux. Les Ho inöhsö:ni' ayant inventé le lacrosse, il leur semblait normal de se voir invités à participer à l'événement. Pour remercier l'Irlande, un collier de wampum à deux rangs a été offerte aux représentants de l'équipe. Le motif des deux ranǵs a pour la première fois été utilisé en 1613 pour sceller l'accord entre les Mohawks et les Hollandais, qui furent autorisés à établir des comptoirs sur le territoire mohawk, le long du fleuve Hudson. L'équipe iroquoise a déclaré à cette occasion: «Vous faites un grand geste, non seulement pour nous mais pour ce qui est juste à vos yeux. Vos actions valent plus que des mots et montrent à tous le vrai pouvoir du sport et l'esprit de lacrosse.»

Les colliers de wampum des Hodinöhsö:ni' sont toujours très vivants aujourd'hui, car nous nous en servons exactement de la même manière qu'il y a deux cents, quatre cents ou mille ans. Ce sont des exemples vibrants d'un patrimoine culturel qui a gardé tout son sens. Un grand nombre de colliers de wampum ont récemment été rendus aux Gardiens des Wampums grâce à une loi fédérale américaine encourageant les musées où ils étaient conservés à les restituer. Depuis, nous essayons de nous souvenir et de redécouvrir les messages dont ils sont porteurs. Des amis et des membres de nos communautés ont fabriqué des copies de ces colliers pour nous permettre de les utiliser lors de manifestations publiques. Comme je l'ai dit plus haut, c'est ainsi que nous avons pu utiliser la George Washington Belt lors de la commémoration du traité de Canandaigua.

Le wampum peut également être utilisé dans un cadre sacré. Dans le contexte de notre Önǵwe'öwehka:a', c'est-à-dire notre mode de vie, le wampum s'inscrit dans un protocole qui en fixe l'usage et le sens. Au sein de nos communautés, certains hommes sont responsables de faire respecter le protocole, par exemple lorsqu'il s'agit de lancer une invitation officielle à un rassemblement. Dans ce cas, le wampum sert à indiquer qu'un «vrai message » est délivré par un «coureur», qui se rend dans la communauté invitée. La lanǵue qui est alors parlée est une des langues hodinöhsö:ni', la formulation étant très importante afin d'éviter tout malentendu. Une fois l'invitation transmise, le destinataire se doit de répondre en suivant les mêmes règles.

Il faut également évoquer le wampum One Dish One Spoon que les Iroquois ont amené avec eux lors 


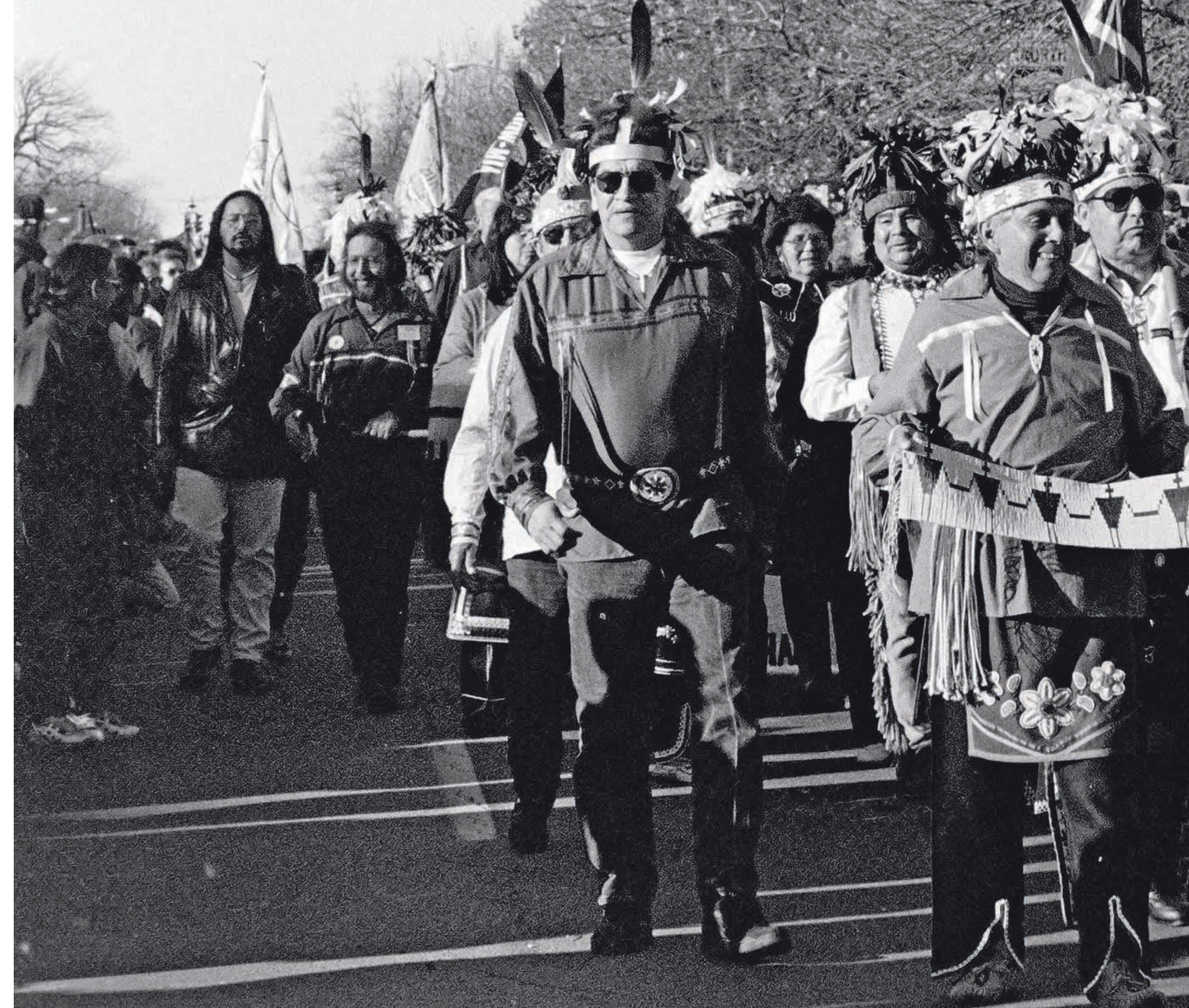




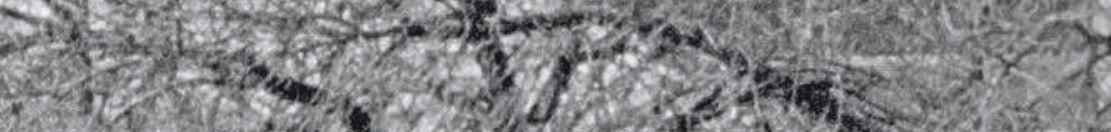

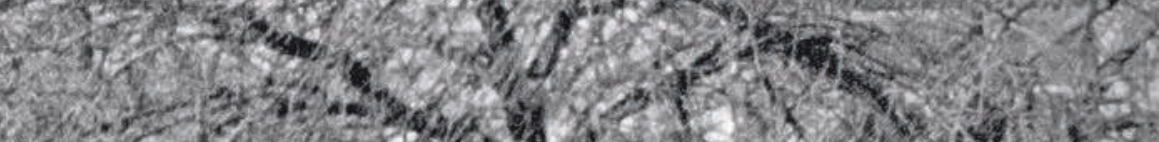

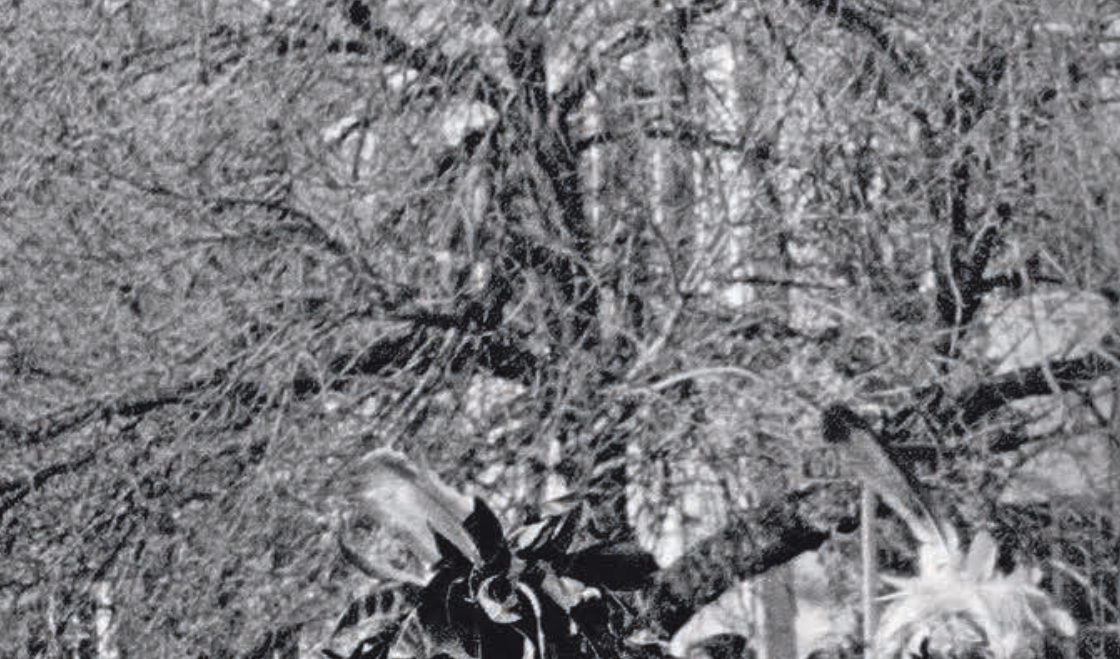

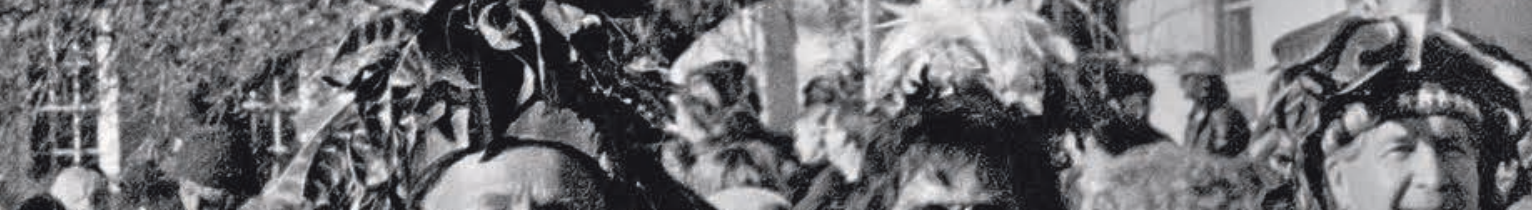

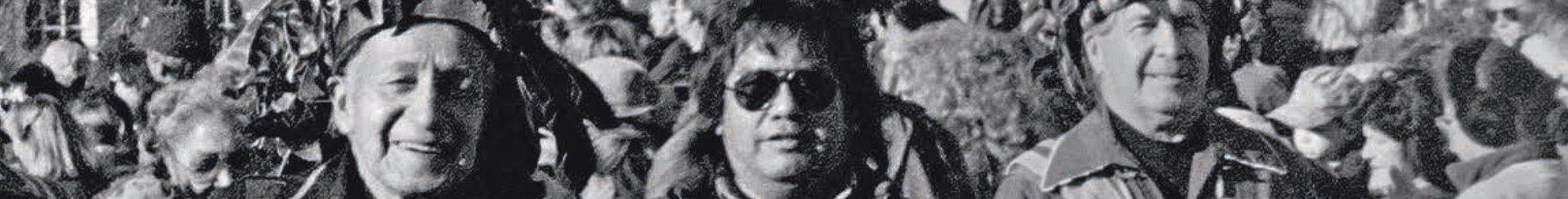

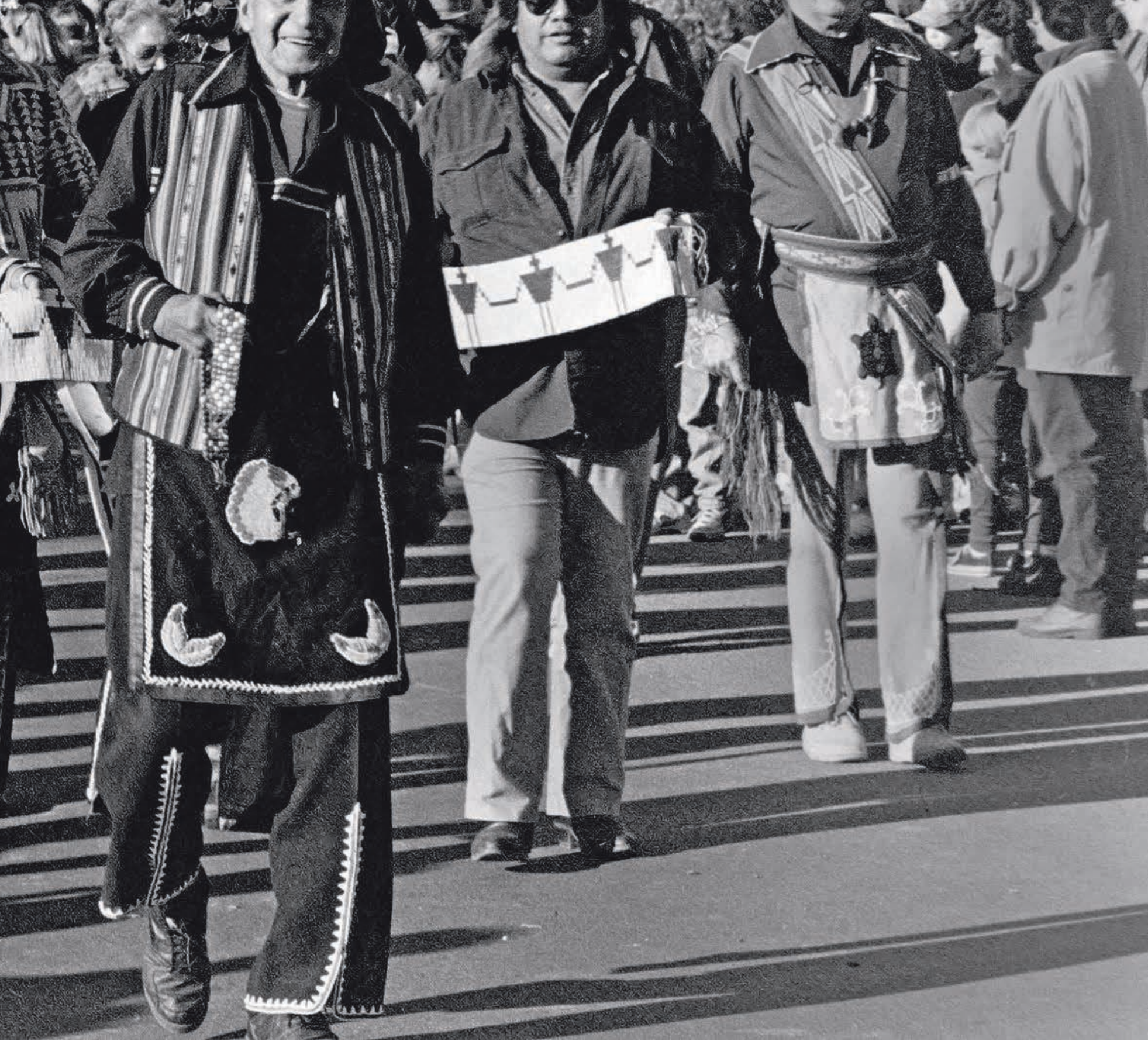




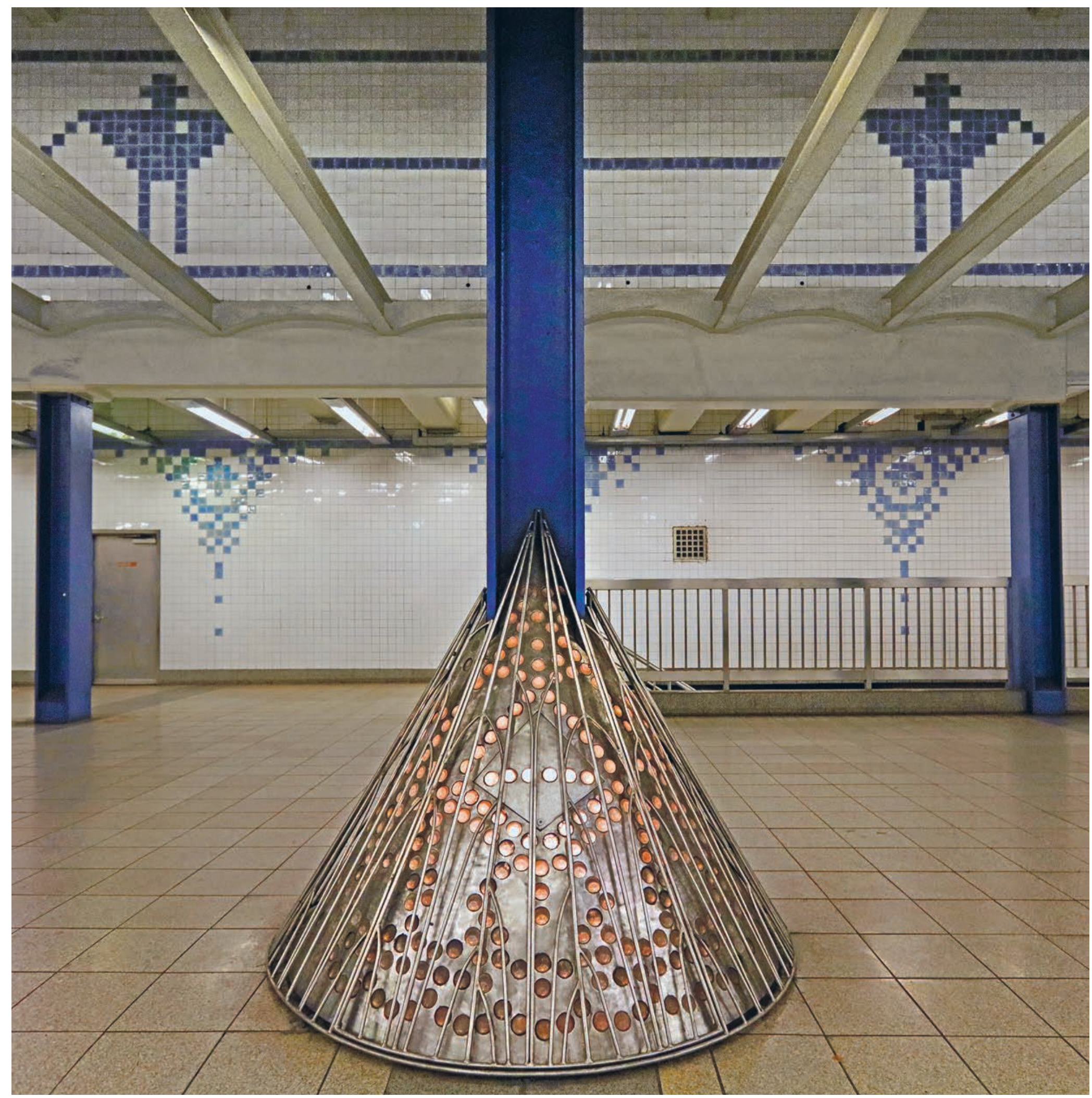

fig. 3. Mel Chin, en collaboration avec Peter Jemison, Signal, 1997. Installation à la station de métro Broadway-Lafayette Street, New York. Commande du Metropolitan Transportation Authority Arts \& Design. Photo MTA Arts \& Design/Rob Wilson. Avec l'aimable autorisation de l'artiste/Thomas Rehbein Galerie, Cologne. 
de leur visite en Israël en 2019, marquée par une série d'actions en faveur de la paix et la réconciliation. Ils ont orǵanisé des stages de lacrosse destinés à des jeunes Israéliens et Palestiniens, réunis à cette occasion. Ils ont partagé la pratique de ce sport, qui est un don du Créateur et est aujourd'hui joué dans le monde entier.

J'ai créé en 1993 avec l'artiste Mel Chin un motif de collier de wampum pour la Metropolitan Transit Authority de la Ville de New York, qui orne les murs de la station de métro Broadway-Lafayette. Le motif provient d'un rêve qui m'a été lu par un membre de la Warm Springs Reservation ${ }^{3}$, en Oregon. L'œuvre a été réalisée avec l'autorisation de feu Leon Schenandoah, un Tadodahoh ${ }^{4}$. Celui-ci était Chef des Gardiens du Feu, la nation Onondaga étant responsable du Feu Central ${ }^{5}$ pour tous les Hodinöhsö:ni'. On y voit six personnages, les bras tendus, reliés entre eux : ils représentent l'attachement aux traditions hodinöhsö:ni', particulièrement importantes dans cette époque difficile marquée par plusieurs tentatives de négociations entre la nation Seneca et l'État de New York, afin que nos droits souverains en tant que nation Seneca soient respectés. Cette création murale a maintenant vingt-huit ans, mais elle conserve selon moi encore toute sa puissance

\section{LE WAMPUM GOMME MÉTAPHORE DE PAIX - MICHAEL GALBAN}

Les perles du wampum remplissent de nombreuses fonctions rituelles chez les les Hodinöhsö:ni'. Ces derniers ont toujours fait usage d'objets matériels pour exprimer des idées et des concepts. L'histoire orale nous dit que, dans les temps anciens, on utilisait à la place des perles de wampum des plumes d'aiǵle et des rameaux de sureau (Gibson 1992). Selon l'histoire du Pacificateur, qui unifia les cinq nations des Hodinöhsö:ni', les coquilles étaient enfilées sur des fils destinés à «élever» et à «redresser» l'esprit de Hiawatha, qui pleurait la perte de sa famille.

En retour, Hiawatha devint l'assistant du Pacificateur. Avec l'aide d'une femme appelée «Mère des Nations », ils établirent la paix entre les cinq nations originelles, qui dura pendant des siècles (Wallace 1968). On confère donc aux perles le pouvoir de guérir et d'apporter de la lumière à une personne ou à une communauté, voire à une nation.

La palourde vit dans les eaux bordant la côte nordest de l'Amérique du Nord. C'est un animal bivalve, dont seule une petite partie de la coquille permet de fabriquer les perles violet foncé. L'autre coquillage utilisé est le bulot. Différentes espèces permettent d'obtenir des perles blanches, mais Sinistrofulgur perversum est la plus courante. Ce gastéropode est unique car les spires de la coquilles présentent la particularité de s'enrouler dans le sens inverse des aiguilles d'une montre, contrairement aux autres espèces de bulots. Dans la culture hodinöhsö:ni', la préférence va souvent aux mouvements qui s'effectuent dans le sens inverse des aiguilles d'une montre, notamment dans les danses. Toutes les danses sociales hodinöhsö:ni' et une grande partie des danses rituelles privilégient des déplacements vers la gauche. On peut également remarquer que le bulot s'attaque aux bivalves comme la palourde. Il utilise la force de son «pied» pour ouvrir le bivalve et extraire l'animal à l'aide de son proboscis. Qu'il s'agisse d'une allégorie ou d'un phénomène naturel, on peut dire que la créature du «coquillage blanc» consomme la créature du «coquillage violet» : c'est là une métaphore de la paix.

L'un des premiers à avoir documenté la culture hodinöhsö:ni' est le père Lafitau, qui consigna ses nombreuses observations des traditions de ce peuple au début du XVIII ${ }^{\mathrm{e}}$ siècle. Il passa une grande partie de sa vie en Amérique du Nord parmi les Kahnawake de Mohawk, mais il mentionne dès le début de son ouvrage : "[C']est, dis-je, dans le commerce de ce vertueux Missionnaire [père Julian Garnier] avec qui j'étois très-étroitement lié, que j'ai comme puisé tout ce que j'ai à dire ici des Sauvages. » (Lafitau 1724) Durant sa carrière de missionnaire, Garnier vécut parmi les Oneida, les Ondondaga et les Seneca. Lafitau put constater la place importante du wampum dans la culture Hodinöhsö:ni' et notamment sa valeur comme mode sacré de communication, mais aussi son rôle rituel fondamental dans le commerce avec des étrangers:

Ils ne croyent pas qu'aucune affaire puisse se déterminer sans ces sortes de Colliers. Quelque proposition qu'on leur fasse, ou quelque réponse qu'on leur donne seulement de bouche, l'affaire tombe, disent-ils, \& ils la laissent effectivement tomber, comme s'il n'en eut jamais été question.

$$
\text { (ibid.: 507) }
$$

Cependant, il est difficile de faire comprendre aux colons européens l'importance du wampum, et Lafitau estime que c'est parce que les Européens ne savent pas répondre avec des colliers de wampum que les Hodinöhsö:ni' se mettent à limiter la taille, la quantité et la qualité de ceux-ci lorsqu'ils échangent avec eux. On retrouve encore aujourd'hui des signes de cette incompréhension, les Hodinöhsö:ni' devant constamment expliquer le rôle culturel des wampums.

\section{IL LES CONDUIT PAR LE BRAS. USAGE DU WAMPUM AUJOURD'HUI - JAMIE JACOBS}

Le terme seneca pour désigner un «wampum d'invitation» est Yötnëshadiyödahǵwa' (yot nes ha dee yon da ǵwa). On peut le traduire par «elle/il l'utilise pour tirer le bras ». La langue iroquoise est relativement unique dans la mesure où les verbes et les noms peuvent être soudés pour former un seul mot. La personne qui fait l'action, ou le sujet, peut être ajoutée à ce mot par un suffixe aspectuel. On obtient ainsi un mot très descriptif qui se traduit par une
3. Territoire des nations Wasco et Paiute dans I'Oregon.

4. Tadodahoh est un titre de chef héréditaire des Onondaga.

5. Chaque nation de la Ligue iroquoise avait son propre feu du conseil, un lieu où un feu était allumé avant que les chefs n'engagent leur discussion sur les sujets touchant la nation. En sus, les Cinq puis Six Nations iroquoises avaient un feu communal à Onondaga, la capitale de la Ligue. C'est dans ce lieu que se rencontraient les cinquante chefs pour discuter des décisions importantes et qu'était conservé le wampum communautaire, sous la esponsabilité des chefs d'Onondaga (Hale 1881: 8 sqq. ; Fenton 1998: 61 sqq.). 
phrase entière en anǵlais ou en français. Cela oblige celui qui écoute à être plus concentré et à réfléchir plus rapidement au contenu de l'énonciation. «Tirer le bras» renvoie à l'action littérale d'inviter ou de déplacer un individu d'un endroit à un autre. Les Hodinöhsö:ni' se sont toujours exprimés par des métaphores qui doivent être interprétées par celui qui écoute, s'il veut comprendre ce qui est «dit vraiment». Jean de Brébeuf, un missionnaire jésuite, a ainsi décrit au XVII ${ }^{\mathrm{e}}$ siècle la langue iroquoise :

Il est vray que leurs discours sont d'abord difficiles a entendre, à cause d'one infinité de Metaphores, de plusieurs circonlocutions, \& autres façons figurées: par exemple parlant de la Nation des Ours, ils diront, l'Ours a dit, a fait cela ; l'Ours est fin, est meschant; les mains de l'Ours sont dangereuses: quand ils parlent de celuy quifait Ie festin des Morts, ils disent, celuy qui mange les ames: quand ils parlent d'one Nation, ils n'en nomment souuent que le principal Capitaine: comme parlant des Montagnets [Montagnais], ils diront, Atsirond dit: c'est le nom d'on des Capitaines. Bref, c'est en ces lieux où ils releuẽt leur stile, $\&$ taschẽt de bien dire. Quasi tous ces esprits sont naturellemẽt d'one assez bonne trempe, ratiocinent fort bien, \& ne brochent point en leurs discours; aussi font-ils estat de se mocquer de ceux qui bronchent: quelques ons semblent estre nés à l'eloquence.

(Thwaites 1897 [1636])

C'est là une déclaration particulièrement forte, quand on sait que la plupart des jésuites savaient lire et écrire les différentes langues d'Europe. Ils savaient également lire et écrire le latin, et certainement le français.

Le «bâtonnet d'invitation» est utilisé par les Iroquois depuis des temps immémoriaux, son origine se perdant dans la nuit des temps. Malgré tout, la tradition rapporte que le premier usage rituel du wampum d'invitation fut institué par celui qui rassembla en une confédération les cinq nations originelles, le Pacificateur. Il dit:

Quand la Nation Onondaga appellera les quatre nations membres à un Grand Conseil, un petit brin de wampum sera porté et délivré par un coureur aux Chefs ou Sachem de la nation pour les inviter au grand rassemblement. Nous appellerons (listerons) le nombre de sommeils durant lesquels le rassemblement aura lieu, et nous les marquerons par des encoches dans un bâtonnet auquel le wampum sera attaché. Ceci sera à respecter afin que le Créateur sache que son peuple assume les responsabilités qu'il a confiées à ces nobles chefs ${ }^{6}$.

Cette tradition est toujours pratiquée aujourd'hui. La nation Onondaga continue d'envoyer des hommes appelés «coureurs» aux chefs des nations, qui sont désormais au nombre de six. Ils leur délivrent un «bâtonnet d'invitation», prononcent des paroles de remerciement, et annoncent les sujets qui seront débattus une fois les chefs rassemblés autour du feu central. Les «coureurs» sont ainsi nommés parce qu'avant l'âge de l'automobile, les hommes devaient réellement courir pour aller voir chaque chef. Arrivés à destination, ils attendaient à l'orée des bois près des ronciers. Ils faisaient un feu et laissaient la fumée monter dans le ciel pour annoncer leur arrivée en attendant d'être escortés dans le village. Les chefs désignaient alors deux jeunes hommes pour aller à leur rencontre et les conduire sans encombre devant le conseil. Un discours d'ouverture était prononcé pour annoncer le motif du rassemblement et «brosser» les coureurs de la tête aux pieds. L'expression «brosser» désigne le fait d'enlever tous les chardons restés accrochés aux coureurs ainsi que les cailloux qui s'étaient collés à leurs pieds pendant leur périple. Pour conclure cette série de gestes de compassion, on leur offrait de l'eau fraîche afin d'apaiser leur gorǵe avant les discours. Une fois encore, ce rituel était autrefois réalisé de manière très littérale par nos ancêtres. Aujourd'hui, le discours a été conservé mais pas l'acte de balayer le corps des coureurs. Une fois que ces derniers se sont rafraîchis et désaltérés, ils prononcent leur discours et les paroles qu'ils ont reçu l'instruction de dire à la nation invitée. Ils commencent par s'adresser aux chefs et aux mères des clans. Ils leur disent que les chefs du village dont ils viennent leur transmettent leurs salutations et espèrent que ce message les trouve en bonne santé. Puis ils s'adressent aux chefs de cérémonie qu'on appelle «Gardiens de la Foi » et leur disent là encore que les «Gardiens de la Foi » de leur village leur envoient leurs salutations et espèrent que ce message les trouve en bonne santé. Vient ensuite le groupe des hommes et des femmes qui n'assument aucune responsabilité ou obligation rituelle. On s'adresse aux enfants en dernier. Une fois que les différents groupes du village ont reçu leur message, l'orateur annonce le nombre de nuits ou de sommeils nécessaires à la nation invitée pour rejoindre le Feu Central du Grand Conseil. Aujourd'hui, on donne souvent le mois et le jour précis, mais on parlait traditionnellement en nombre de nuits. Les encoches faites dans le bâtonnet correspondent à ce nombre de nuits. On rapporte que, «dans les temps anciens », dès qu'une nuit était passée, on brisait le bâtonnet à l'endroit d'une encoche jusqu'à la dernière; au stade de la dernière encoche la nation invitée se devait d'avoir rejoint le territoire de la nation Onondaǵa pour la tenue du conseil. Une fois que les discours ont été prononcés, la nation invitée demande aux coureurs de se mettre de côté afin de leur soumettre en privé des questions ou des problèmes qui méritent d'être débattus. Si tout 


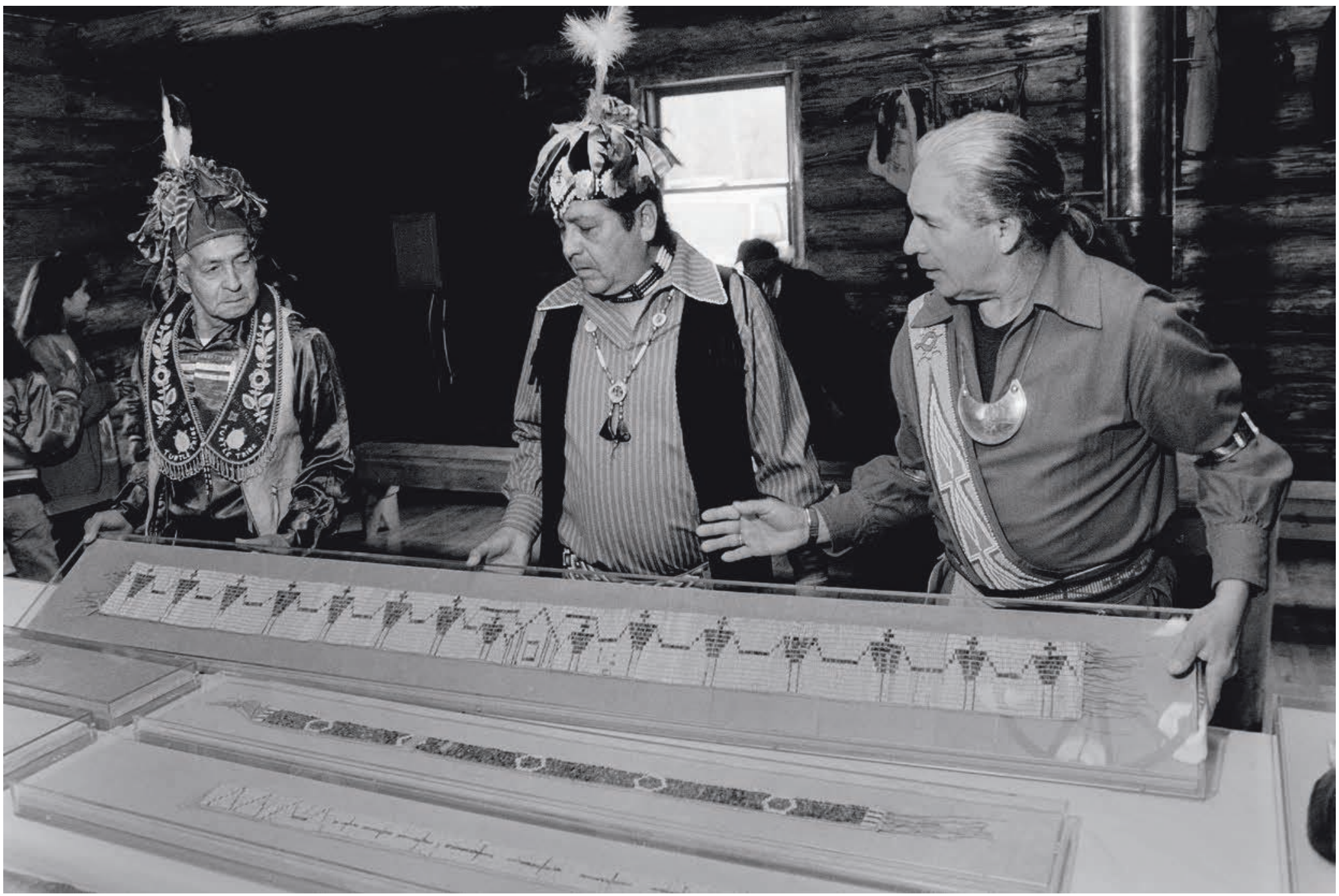

fig. 4. Présentation du collier «Washington Covenant Wampum» dans la maison longue de la Nation Onondaga après sa restitution par le Albany Institute of History and Art en 1989. De gauche à droite: le Tododaho (chef spirituel) Leon Shenandoah, le chef Irving Powless et le Gardien de la Foi Oren Lyons. Photo Mike Greenlar, 1988. Avec l'aimable autorisation de l'artiste. 
Dossier

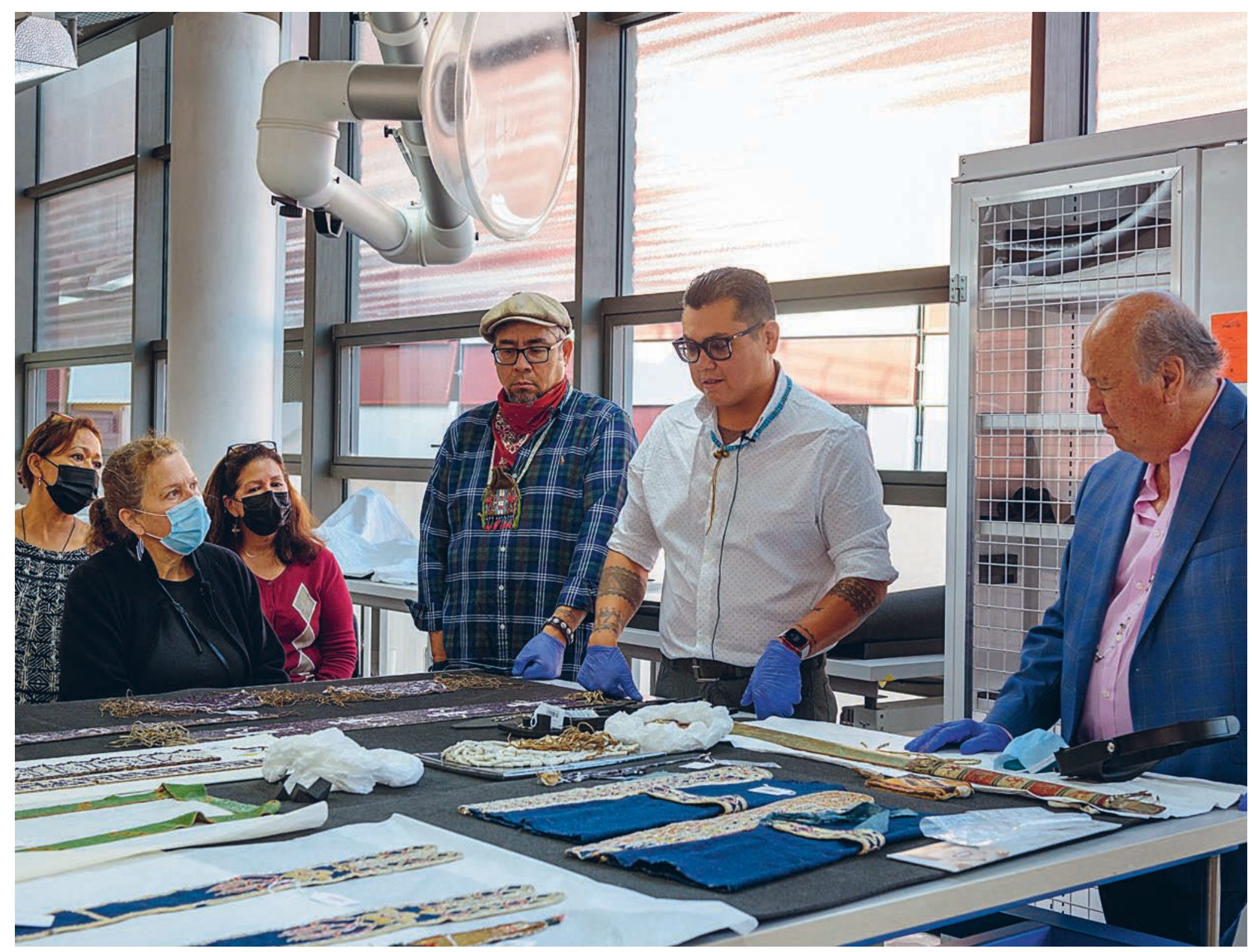

fig. 5. Séance de consultation de la collection de wampums au musée du quai Branly-Jacques Chirac

(de gauche à droite: Katsitsionni Fox, lakonikonriiosta, Tonia Galban, Michael Galban, Jamie Jacobs, Peter Jemison), 13 novembre 2021. Photo Alice Sidone. 
le monde est d'accord sur le message, les coureurs sont rappelés devant le conseil et les nations invitées désignent un orateur qui ira en leur nom porter leurs salutations et dire que le contenu du message est accepté. On prie le «Détenteur des Cieux» que les coureurs rentrent sains et saufs chez eux et on rend grâce pour les traditions culturelles qui survivent. À leur arrivée en territoire onondaga, les nations invitées apportent le bâtonnet d'invitation au rassemblement et un discours est prononcé pour le rendre à la nation hôte. Les mêmes formules sont utilisées, mais à la fin du discours, on remercie le Grand Créateur d'avoir veillé sur le voyage et sur la santé des participants. Une fois que toutes les nations invitées ont procédé de la sorte et que tous les bâtonnets d'invitation ont été rendus, le conseil peut commencer.

Nous avons beaucoup de chance que nos ancêtres aient réussi à maintenir cette tradition vivante et nous leur sommes éternellement reconnaissants d'avoir su la sauvegarder pour nous permettre de la faire vivre

\section{Les auteurs}

Peter Jemison est membre du Clan du Héron de la nation Seneca. Il est Gardien de la Fo de la Newtown Longhouse sur la Cattaraugus Reservation. Peter est aussi responsable du Ganondagan State Historic Site, I'emplacement d'un village seneca du xvII ${ }^{\mathrm{e}}$ siècle situé à Victor, dans I'État de New York. Il est artiste, conservateur, réalisateur et auteur renommé. Représentant de la nation Seneca, il a participé à la restitution de restes humains, d'objets sacrés et de wampums pendant plus de trente ans. Il dirige actuellement le 1794 Canandaigua Treaty Commemoration Committee et organise le 11 novembre de chaque année une commémoration de ce traité dans la ville de Canandaigua, dans I'État de New York.

Michael Galban est issu de la nation Washoe du Nevada et de la Californie et de la nation Yosemite-Mono Lake Paiutes. Il est conservateur du Seneca Art \& Culture Center de Ganondagan, où il travaille depuis trente ans. Ses recherches portent sur la culture matérielle portent sur la culture materi
des Terres boisées de l'Est, notamment sur le peuple Hodinöhsö:ni'. Il estartiste, auteur, historien et père de trois enfants kaniekehake (mohawk).

Hoya'danä:gwad (Jamie Jacobs) est membre du Clan de la Tortue de la nation Seneca de Tonawanda. Il est né et a grandi sur le territoire Tonawanda Seneca. Il est le gardien des rituels pour la Tonawanda Longhouse, et il enseigne également la langue seneca par le biais de programmes d'immersion et de formation des enseignants. II travaille depuis quinze ans pour la Rock Foundation du Rochester Foundation du Rochester
Museum and Science Center. Il étudie aujourd'hui la culture matérielle des Hodinöhsö:ni', en particulier l'archéologie seneca depuis la période précoloniale jusqu'au début du xIx siècle. 


\section{Bibliographie}

\section{Fenton, William N.}

1998 The Great Law and the Longhouse: A Political History of the Iroquois Confederacy. Norman, University of Oklahoma Press.

\section{Gibson, John Arthur}

1992 [1912] Concerning the League: The Iroquois League Tradition as Dictated in

Onondaga, trad. de l'onontagué et éd. par Hanni Woodbury et al. Winnipeg, Algonquian and Iroquoian Linguistics : 328-329.

\section{Hale, Horatio}

1881 Hiawatha and the Iroquois confederacy: A study in anthropology. Salem (Massachusetts), Salem Press.

\section{Lafitau, Joseph-François}

1724 Mours des sauvages amériquains, comparées aux mours des premiers temps.

Paris, Sauǵrain l'aîné/

Hochereau, t. I : 3.

Thwaites, Reuben Gold (éd.)

1897 (1636) The Jesuit

Relations and Allied Documents

Travels and Explorations

of the Jesuit Missionaries in New France, 1610-1791: The Original French, Latin, and Italian Texts, with Enǵlish Translations and Notes, t. X: Relation de ce qui s'est passé en la Nouvelle France en l'année 1636 - Jean de Brébeuf. Cleveland (Ohio), Burrows Brothers : 256 et 258 .

Wallace, Paul Anthony Wilson 1968 The White Roots of Peace. Port Washinǵton, Friedmann. 


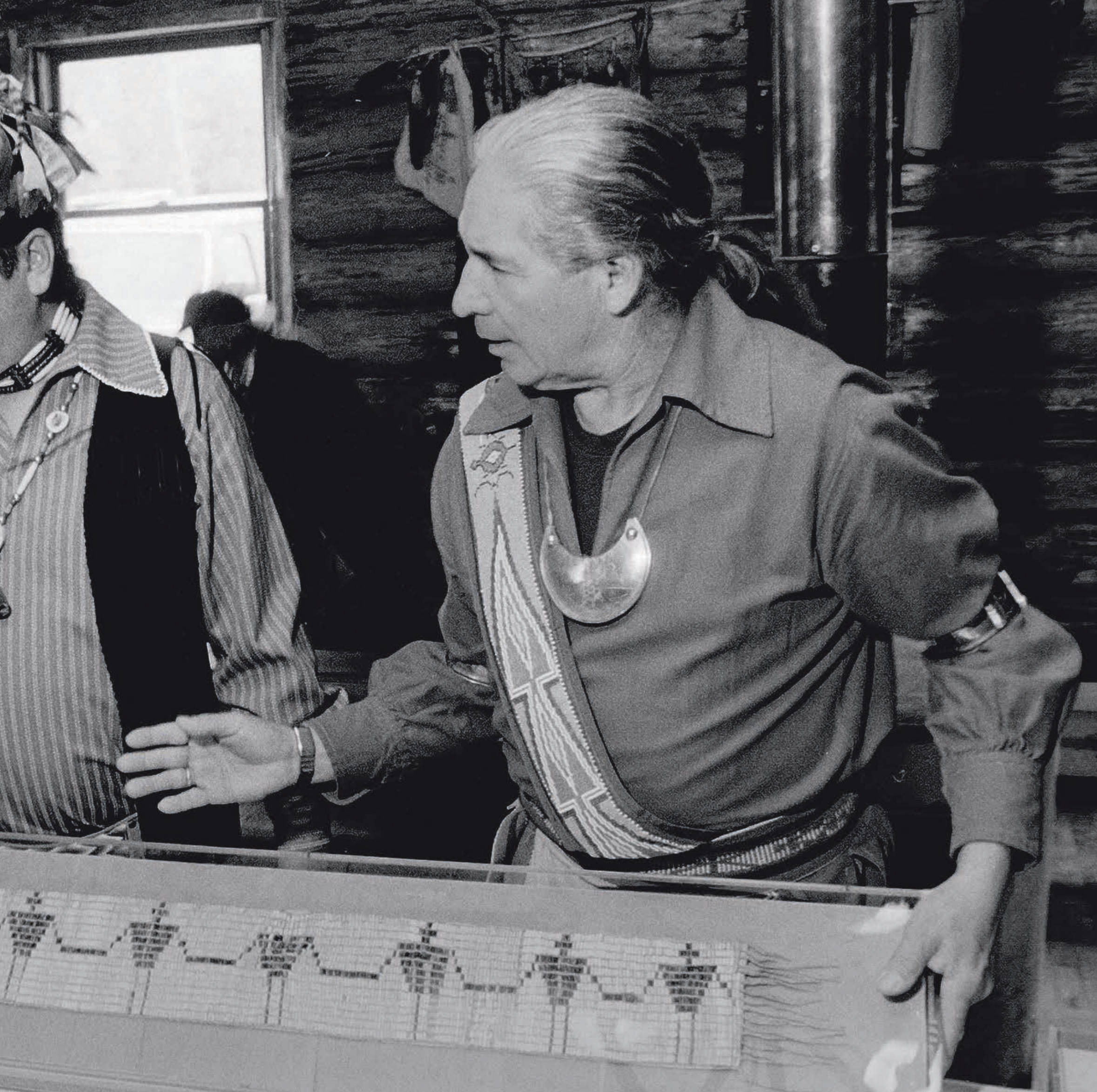

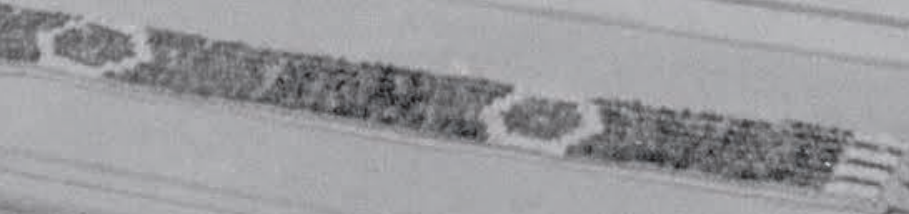

